\title{
VLBA images of the precessing jet of LS I $+61^{\circ} 303$
}

\author{
M. Massi ${ }^{1}$, E. Ros ${ }^{2,1}$, and L. Zimmermann ${ }^{1}$ \\ ${ }^{1}$ Max-Planck-Institut für Radioastronomie, Auf dem Hügel 69, 53121 Bonn, Germany \\ e-mail: [mmassi; lzimmerm] @mpifr-bonn.mpg.de \\ 2 Departament d'Astronomia i Astrofísica, Univ. de València, 46100 Burjassot, Valencia, Spain \\ e-mail: Eduardo.Ros@uv.es
}

Received 13 September 2011 / Accepted 20 February 2012

\section{ABSTRACT}

\begin{abstract}
Context. In 2004, changes in the radio morphology of the Be/X-ray binary system LS I $+61^{\circ} 303$ suggested that it is a precessing microquasar. In 2006, a set of VLBA observations performed throughout the entire orbit of the system were not used to study its precession because the changes in radio morphology could tentatively be explained by the alternative pulsar model. However, a recent radio spectral index data analysis has confirmed the predictions of the two-peak microquasar model, which therefore does apply in LS I $+61^{\circ} 303$.

Aims. We revisit the set of VLBA observations performed throughout the orbit to determine the precession period and improve our understanding of the physical mechanism behind the precession.

Methods. By reanalyzing the VLBA data set, we improve the dynamic range of images by a factor of four, using self-calibration. Different fitting techniques are used and compared to determine the peak positions in phase-referenced maps.

Results. The improved dynamic range shows that in addition to the images with a one-sided structure, there are several images with a double-sided structure. The astrometry indicates that the peak in consecutive images for the whole set of observations describes a well-defined ellipse, 6-7 times larger than the orbit, with a period of about $28 \mathrm{~d}$.

Conclusions. A double-sided structure is not expected to be formed from the expanding shocked wind predicted in the pulsar scenario. In contrast, a precessing microquasar model can explain the double- and one-sided structures in terms of variable Doppler boosting. The ellipse defined by the astrometry could be the cross-section of the precession cone, at the distance of the $8.4 \mathrm{GHz}$-core of the steady jet, and $28 \mathrm{~d}$ the precession period.
\end{abstract}

Key words. radio continuum: stars - X-rays: binaries - X-rays: individuals: LS I $+61^{\circ} 303$ - gamma rays: stars - astrometry galaxies: jets

\section{Introduction}

In 1993, a VLBI observation of LS I $+61^{\circ} 303$ showed that the radio emission had a structure of milliarcsecond (mas) size corresponding to a few $\mathrm{AU}$ at the distance of $2.0 \mathrm{kpc}$ (Massi et al. 1993; Frail \& Hjellming 1991). The complex morphology in successive VLBI observations (Peracaula et al. 1998; Paredes et al. 1998; Taylor et al. 2000; Massi et al. 2001, 2004) highlighted the unlikelihood of an interpretation in terms of a microquasar with a constant position angle. The radio morphology not only changes position angle, but it is even sometimes onesided and at other times two-sided. This suggested the hypothesis of LS I $+61^{\circ} 303$ being a precessing microblazar (Kaufman Bernadó et al. 2002; Massi et al. 2004). A microblazar, i.e., a microquasar with radio jets forming a small angle, $\theta$, with respect to the observer's line of sight, has been proposed to be the Galactic version of extragalactic blazars. Doppler boosting enhances the radiation from material that is moving towards the observer, and attenuates it when it moves in the opposite direction. In cases of substantial flux attenuation in the receding jet (i.e., attenuation to a level fainter than the sensitivity of radio images), the structure will appear as a one-sided jet. A precession of the jet leads to a variation in the angle, $\theta$, and therefore variable Doppler boosting. The result is both a continuous variation in the position angle of the radio-emitting structure and of the flux density ratio of the approaching to the receding jet (Massi 2007).

In known precessing $\mathrm{X}$-ray binaries, the timescale for tidally forced precession of the accretion disk around the compact object, induced by the companion star, lies within the range 8-22 times the orbital period (Larwood 1998; Massi \& Zimmermann 2010). In this context, the peculiarity of the variations in LS I $+61^{\circ} 303$ is their short timescale with respect to the orbital period of $26.496 \mathrm{~d}$. Massi et al. (2004) found that MERLIN images revealed a surprising variation of $60^{\circ}$ in position angle in only one day. Even if quantitatively the relationship between position angle in the image and the viewing angle $\theta$ is not straightforward, a rapid variation in the position angle implies nevertheless that there is clearly a rapid variation in $\theta$. The fast position angle variation has been confirmed by VLBA observations. Dhawan et al. (2006) measured in VLBA images a rotation of the inner structure of roughly $5^{\circ}-7^{\circ}$ in $2.5 \mathrm{~h}$, that is again a variation of almost $60^{\circ}$ day, but suggested that the variations were due to a cometary tail of a pulsar. If the compact object is a pulsar, the interaction between its relativistic wind and the equatorial wind of the $\mathrm{Be}$ star is predicted to create a bow-shock around the pulsar with a sort of cometary tail, i.e., a one-sided structure, extending away from the Be star (Dubus 2006). However, the analysis of the radio spectral index by Massi \& Kaufman Bernadó (2009) prove that LS I $+61^{\circ} 303$ displays the typical characteristic of microquasars of an optically thin outburst after an interval of optically thick emission. In microquasars, the so-called transient jet associated with the large optically thin outburst, is related to shocks travelling in a pre-existing steady jet, that is a slow-moving continuous conical outflow with a composite flat/inverted radio spectrum (i.e., optically thick emission) (Fender et al. 2004; Massi 2011). 
The remarkable finding for $\mathrm{LS} \mathrm{I}+61^{\circ} 303$ is that, during the maximum of its long-term periodicity ( $4.6 \mathrm{yr}$ ) the alternance between optically thick and optically thin emission, occurs twice during the orbit, first around periastron and then again, shifted by almost $0.3-0.4$ in orbital phase $(0.3-0.4 \times 26.496 \mathrm{~d}=8-$ $11 \mathrm{~d}$ after periastron), i.e. towards apastron (Massi \& Kaufman Bernadó 2009). This agrees with the well-known "two peak accretion/ejection model", which has been applied by several authors to LS I $+61^{\circ} 303$, predicting, for large mass accretion rate, $\dot{M}$, two events: one around periastron and the second shifted about 0.3 in orbital phase towards apastron (Taylor et al. 1992; Marti \& Paredes 1995; Bosch-Ramón et al. 2006; Romero et al. 2007).

The radio spectral index data corroborate the microquasar model for LS I $+61^{\circ} 303$. The most important uncertainty is therefore the process that could produce the observed fast precession. An essential step in any investigation is to establish the precessional period. This parameter could be derived by the reanalysis of the VLBA observations. These observations were performed by Dhawan et al. (2006) every 3 days over 30 consecutive days, towards the minimum of the long-term periodicity. Dhawan et al. (2006) suggested that the peaks of the maps trace an erratic ellipse. In a precessing microblazar, as explained below, the core component of the steady jet describes an ellipse, whereas the transient jet adds random shifts. To determine important precession parameters, e.g. the period, we therefore reanalyzed the set of VLBA observations performed by Dhawan et al. (2006).

\section{Data reduction and results}

LS I $+61^{\circ} 303$ was observed by the VLBA on ten different days from 2006 June 30 (segment A) to 2006 July 30 (segment J). The observations (code BD117) included data at $2.3 \mathrm{GHz}$ and $8.4 \mathrm{GHz}$. We performed the post-correlation data reduction using AIPS. The phase and amplitude calibration were performed in a standard way: corrections for residual Earth orientation parameters in the correlator model and ionosphere - in the latter case those derived from GPS data - were introduced; the digital sampling amplitude corrections (ACCOR) were applied; system temperature and gains were applied to get the amplitude calibration (APCAL); delay offsets between sub-bands were corrected using pulse-tones measured during the observations (APCOR); a parallactic angle correction was introduced; and the final delay and rate calibration across the observing band was performed using the FRING routine. After these procedures, the data for the phase reference calibrator, J0244+6228, were exported to be imaged in Difmap. We read the image back into AIPS and calibrated the amplitudes and phases for the source and produced a hybrid map of the source with IMAGR within AIPS, that matched the results obtained with Difmap. After that, the delay and rate were calibrated again with FRING after dividing the data by the clean-component model produced by IMAGR for this source to get structure-free solutions for J0244+6228. The structure-free solutions obtained were then interpolated to the target source, $\mathrm{LS} \mathrm{I}+61^{\circ} 303$, and those data were exported to be imaged.

\subsection{Analysis and results of the $8.4 \mathrm{GHz}$ data}

The $8.4 \mathrm{GHz}$ phase-referenced images (with respect to J0244+6228) shown in Fig. 1, closely reproduce the Dhawan et al. (2006) structures. At their center, we show the orbit with a semimajor axis of $(0.22-0.23)$ mas $\left(\simeq 0.7 \times 10^{13} \mathrm{~cm}\right.$ at a distance of $2 \mathrm{kpc}$ ), that was derived from the third Keplerian law for $P=26.496 \mathrm{~d}$ with a mass for the B0 star of $17 M_{\odot}$ and a mass for the compact object of 1.4-4 $M_{\odot}$, and traced using Casares et al. (2005) orbital parameters. The orbit is not only much smaller than the radio-emitting region, but also smaller than the interferometric beam of the VLBA at this wavelength. We make this remark because the zoomed orbit in Fig. 3 by Dhawan and collaborators (zoomed to show more clearly the orbital phase of each run) may unfortunately produce the wrong impression that the orbit is resolved when it is not, and only the accuracy of the astrometry, which is 0.04 mas at $8.4 \mathrm{GHz}$, is of a smaller scale than the orbit (see Sect. 2.2).

For each run, we present in Fig. 1 both the phasereferenced and the self-calibrated images. Self-calibrated images were produced with the automatic procedure Muppet of the Caltech Difmap imaging package (Shepherd 1997). Using self-calibration, the information on absolute flux density and absolute position are lost, but the removal of residual calibration errors improves the dynamic range by up to a factor of four for our maps (Cornwell \& Fomalont 1999). Muppet starts with a point source as an initial model then automatically selects the clean boxes around peaks with signal-to-noise ratio $(\mathrm{S} / \mathrm{N})$ values $\geq 6$, and switches on amplitude plus phase self-calibration after phase-only self-calibration cycles have converged. The high limit of the signal-to-noise ratio $S / N \geq 6$ prevents the risk of creating artifacts, which are only introduced when self-calibrating below $S / N<4$ (Martí-Vidal \& Marcaide 2008). For epoch G, there is a significant difference between the phase-referenced image and the self-calibrated image at the same resolution (Fig. 1). Therefore, for this epoch, we also self-calibrated the data manually. Figure 2 shows an intermediate image after eight manual iterations of hybrid mapping starting with a point source and using phase-only self-calibration. The two images of Fig. 2 clearly show the same structures only with different flux densities. This difference depends upon further iterations of amplitude self-calibration performed by Muppet.

The determination of the peak position in the phasereferenced maps is an issue, especially if the source is not Gaussian-like or symmetric, as was the case in some epochs. The first approach is to determine the location of the brightest pixel with IMSTAT, then three different methods were applied to fit the position either in the image plane with JMFIT and MAXFIT, or in the visibility plane, with modelfit in Difmap. All results from the four applied methods coincide within $(1-3) \sigma$, trace an ellipse over time, and show that the peak of run E is displaced from the other peaks. However, the Gaussian fits have asymmetric residuals, which indicates that the brightest parts of the images are asymmetric. As discussed in Sect. 3, the core of the steady jet for a microblazar is clearly not Gaussian, because of Doppler boosting: the redshifted part should be fitted with a smaller half ellipse than the blueshifted part. For consistency, we use for Fig. 3 the results for IMSTAT (coincident within $1 \sigma$ with MAXFIT). It is evident that the peaks of the consecutive images clearly trace an ellipse 6-7 times larger than the orbit. Galactic scattering at $8.4 \mathrm{GHz}$, as discussed in the next section, gives rise to an error of 0.04 mas. This value is slightly smaller than the average value of 0.05 mas estimated for the astrometric accuracy of a full track phase-referenced VLBI experiment (Pradel et al. 2006).

In the images, the larger dynamic range of the self-calibrated maps show in the image of run A a double-component structure. This is clearly unlikely to correspond to a pulsar nebula, whereas it could be compatible with either the approaching and 
M. Massi et al.: VLBA images of LS I $+61^{\circ} 303$
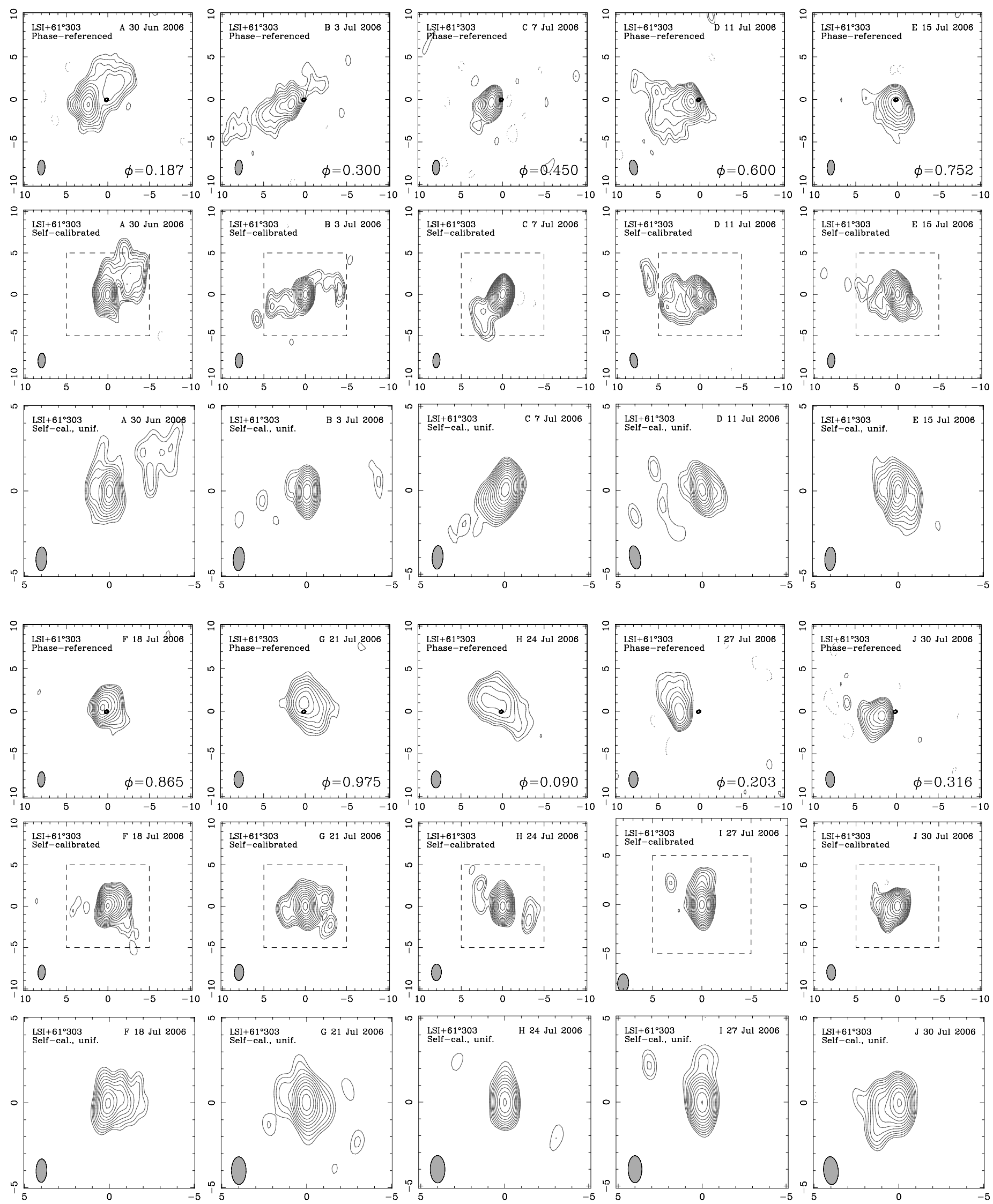

Fig. 1. Images of VLBA runs A-J at $8.4 \mathrm{GHz}(3.6 \mathrm{~cm})$ of LS I $+61^{\circ} 303$. The units on the axes are milliarcseconds (mas). For each run, three maps are presented, the phase-referenced map (beam of 1.8-2.0 mas $\times 0.9-1.1$ mas, shown in the bottom left corner), the self-calibrated map at the same resolution, and the self-calibrated map with a beam of 1.4-1.6 mas $\times 0.6-0.9$ mas. Contour levels for all maps are $-4,4,5.66,8,11.3,16,22.6,32$, $45.2,64,90.5,128,181 \sigma$ (with $1 \sigma=0.1 \mathrm{mJy} /$ beam for the top and bottom maps, $1 \sigma \simeq 0.07$ middle maps). At the center of the phase-referenced images, we trace, in scale, the orbit (see Sect. 2). 

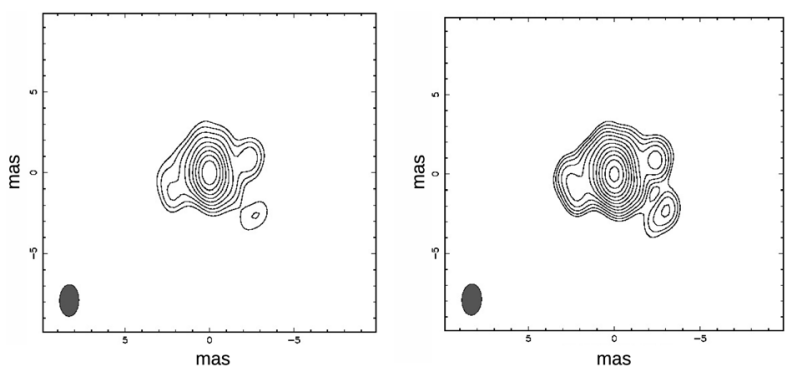

Fig. 2. Left: intermediate self-calibrated image of run $\mathrm{G}$, at $8.4 \mathrm{GHz}$, after eight manual iterations of hybrid mapping with phase-only selfcalibration. The beam is 1.96 mas $\times 1.14$ mas at $-2^{\circ}$. Contour levels are $-4,4,5.66,8,11.3,16,22.6,32,45.2,64 \sigma$, with $1 \sigma=$ $0.18 \mathrm{mJy} /$ beam. Right: output of automatic hybrid mapping by Muppet. Muppet switches on amplitude plus phase self-calibration after phaseonly self-calibration cycles have converged. The beam is 1.96 mas $\times$ 1.14 mas at $1^{\circ}$. Contour levels are $-4,4,5.66,8,11.3,16,22.6,32$, $45.2,64,90.5,128,181 \sigma$, with $1 \sigma=0.08 \mathrm{mJy} /$ beam.

receding component of a transient jet, or with variable Doppler boosting along a one-sided twisted jet. Image $\mathrm{B}$ already shows in the phase-referenced map with natural weighting a doublesided morphology difficult to reconcile with the pulsar nebula. The self-calibrated map shows an intriguing twisted jet as expected for a fast precession. Runs C, F, I, and J show a one-sided jet or alternatively a pulsar cometary-tail but again runs D, E, G, and $\mathrm{H}$ show the double-sided jet structure.

\subsection{Analysis and results of the $2.3 \mathrm{GHz}$ data}

In Fig. 4 of Dhawan et al. (2006), the $2.3 \mathrm{GHz}$ astrometry seems to show a shift of up 2 mas from the $8.4 \mathrm{GHz}$ astrometry. In practice, no hypothesis can be made lacking the points of error bar. We show in this section that, whereas the accuracy associated with the peaks at $8.4 \mathrm{GHz}$ is 0.04 mas, that associated with the peaks at $2.3 \mathrm{GHz}$ is more than one order of magnitude higher.

We re-examined the $2.3 \mathrm{GHz}$ data. Large amounts of Galactic scattering at this frequency affects the data, broadening the images. This effect is discussed in detail in the astrometric works of Lestrade and collaborators (Lestrade et al. 1985, 1999), who attributed the size of 4.1 mas, determined at $1.62 \mathrm{GHz}$, to broadening caused by interstellar scattering. Therefore, at $2.3 \mathrm{GHz}$ the broadening, which is proportional to $\lambda^{2}$, is 2.2 mas (whereas at $8.4 \mathrm{GHz}$, it is only 0.16 mas). The additional complicating factor is that refractive interstellar scintillation will cause the apparent source position to wander, and the magnitude of this wandering is hard to calculate (see, e.g. Rickett 1990).

We were able to estimate the quality of the astrometry from the scatter in positions of the check source observed in project BD117, namely $\mathrm{J} 0239+6005$, that is $2.5^{\circ}$ apart from the calibrator J0244+6228. We determined the astrometric stability of that check source at $8.4 \mathrm{GHz}$ and $2.3 \mathrm{GHz}$ and used the results to assign error bars to the astrometric measurements at both frequencies. First, we performed the phase-referencing analysis of the source J0239+6005 with respect to the phase reference calibrator J0244+6228, and then determined the peak position in the maps of J0239+6005 for the consecutive runs. As shown in Fig. 4, when there is no precession and one expects all results to coincide within their errors, the points at $8.4 \mathrm{GHz}$ indeed overlap and have a dispersion of 0.08 mas. The result at low frequency is completely different. The scatter is huge: the dispersion in the points at $2.3 \mathrm{GHz}$ is 1.2 mas. The astrometric error scales with separation from the phase reference calibrator

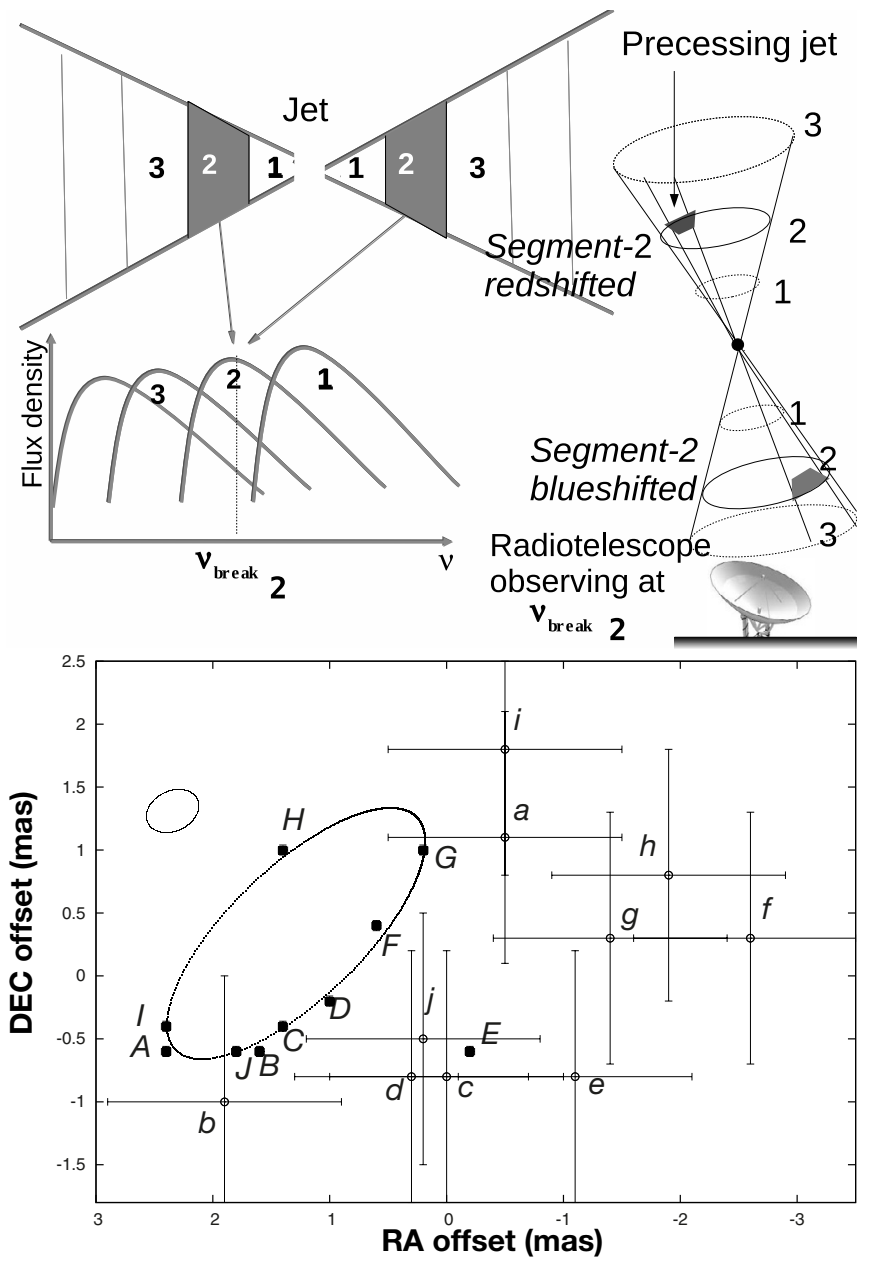

Fig. 3. Top-left: superposition of individual spectra, each with a different $v_{\text {break }}$, associated with different segments of a steady jet. Top-right: for a precessing microblazar, the core component, dominated by the approaching jet contribution because of Doppler boosting, will describe an ellipse during precession. Bottom: astrometry of consecutive peaks of VLBA $8.4 \mathrm{GHz}$ maps (capital letters) and $2.3 \mathrm{GHz}$ maps (small letters) for runs A-J with the orbit drawn to scale at an arbitrary distance. East is to the left. Peak of $8.4 \mathrm{GHz}$ map E, even if displaced from the other ones, which is therefore likely affected by the approaching component of the transient jet, is at a position angle that is consistent with the other peaks. The small error of 0.04 mas for the astrometry at $8.4 \mathrm{GHz}$ is indicated by the filled squares. That the precessing compact object moves in an orbit with semimajor axis of $\sim 0.2$ mas introduces additional variations. Errorbars of 1 mas for the astrometry at $2.3 \mathrm{GHz}$ represent only a lower limit to the error associated with large Galactic scattering and low resolution (see Sect. 2.2).

(Pradel et al. 2006). J0239+6005 is $2.5^{\circ}$ apart from the calibrator J0244+6228, whereas LS I $+61^{\circ} 303$ is $1.4^{\circ}$ away from $\mathrm{J} 0244+6228$. Therefore, the 0.08 mas rms for $8.4 \mathrm{GHz}$ data and the 1.2 mas rms for $2.3 \mathrm{GHz}$ data of J0239+6005 scales to 0.04 mas and 0.7 mas, respectively, for $\mathrm{LS} \mathrm{I}+61^{\circ} 303$. Moreover, at $2.3 \mathrm{GHz}$, besides the already large error of 0.7 mas associated with the scattering, there is also the error due to the low resolution. In fact, at $2.3 \mathrm{GHz}, \mathrm{LS} \mathrm{I}+61^{\circ} 303$ is unresolved/barely resolved with the VLBA (see Fig. 5). In the large beam, 4 mas $\times$ 7 mas, the optically thin emission of the extended jet may contribute substantially and, depending on its brightness, displace the peak out of the core position, especially in the case of onesided structures. At some low level, we can test this effect with the data at $8.4 \mathrm{GHz}$, in a similar way as done in Fig. 4 by 


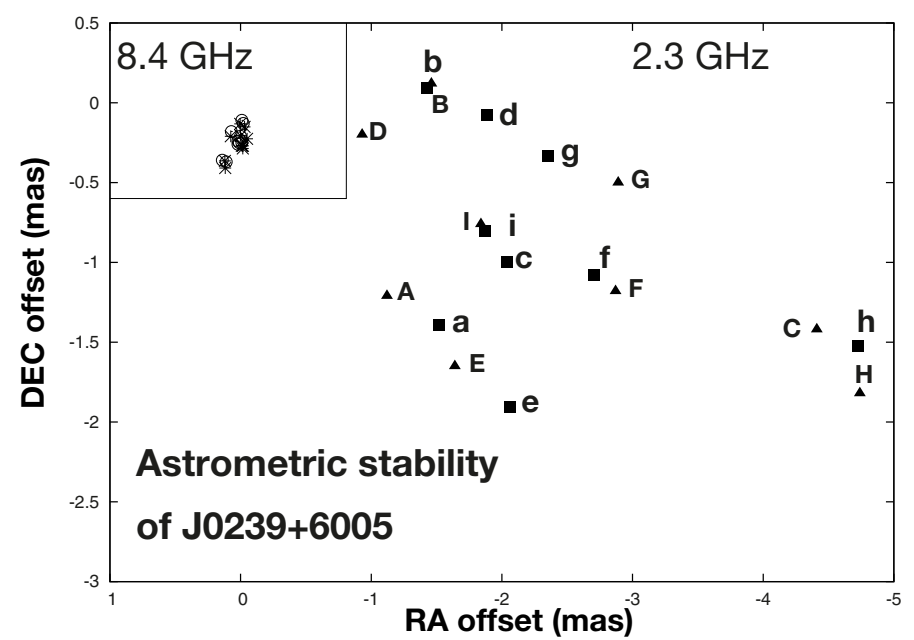

Fig. 4. Control astrometry on the secondary calibrator. Relative positions of $\mathrm{J} 0239+6005$ with respect to $\mathrm{J} 0244+6228,2.5^{\circ}$ apart. The astrometry at $2.3 \mathrm{GHz}$ is indicated by squares (JMFIT) and triangles (MAXFIT); that at $8.4 \mathrm{GHz}$ is indicated by crosses (JMFIT) and circles (MAXFIT)

Dhawan et al. (2006) where one can see peak and "centroid" positions for $3.6 \mathrm{~cm}$ (i.e. $8.4 \mathrm{GHz}$ ). We strongly tapered the data at $8.4 \mathrm{GHz}$ to reproduce the large beam at $2.3 \mathrm{GHz}$. We then fitted the peak positions in the low resolution maps obtained and compared them with those fitted in the maps of Fig. 1 (i.e. the points along the ellipse of Fig. 3). This test at $8.4 \mathrm{GHz}$, which can obviously not take into account resolved larger structures, measures differences between positions with a dispersion of 0.6 mas. This error adds quadratically to the error of about 0.7 mas associated with the scattering, resulting in the total error of $\sim 1$ mas for the astrometry of LS I $+61^{\circ} 303$ given in Fig. 3-bottom. We note that with this value, which represents, however, only a lower limit to the error bars, there is an overlap at each epoch at $2.3 \mathrm{GHz}$ of the results from the different fitting programs, which can differ by up to 2 mas.

We note that the $2.3 \mathrm{GHz}$ astrometry has a general shift with respect to that at $8.4 \mathrm{GHz}$. This, as discussed in the next section, would correspond to the predictions of the core-shift effect. Owing to this effect, the peak of the emission (i.e. core) representing the optically thick part of the jet at one observing frequency, is predicted to have a shift from the center that is different for the different frequencies (Fig. 3-top). This shift can also be seen in the $2.3 \mathrm{GHz}$ astrometry of Dhawan et al. (2006) without the error bars. As in our case, Dhawan et al. ascribed the shift to a synchrotron opacity gradient along the emitting structure. However, in that case the structure is not thought to be a precessing jet but a cometary tail. Anything more than a general trend cannot be inferred from the $2.3 \mathrm{GHz}$ data. As a result of our analysis of the data at $2.3 \mathrm{GHz}$, it is indeed clear that any quantitative comparison between the astrometry at $8.4 \mathrm{GHz}$ and at $2.3 \mathrm{GHz}$ (as well as between the orientation of the radio structures at the two frequencies) is unfortunately prevented by the very large Galactic scattering and the low resolution.

\section{Precessing microblazar}

Figure 3-bottom shows the results of the astrometry for the $v=8.4 \mathrm{GHz}$ data: the peaks of the consecutive images clearly trace an ellipse. Here, we show that this ellipse could be the cross-section of the precession cone, at the distance of the $3.6 \mathrm{~cm}$-core $(v=8.4 \mathrm{GHz})$ of the steady jet.
A magnetized plasma containing energetic electrons with a power-law energy distribution will produce a synchrotron power-law spectrum. However, below a critical frequency $\left(v_{\text {break }}\right)$, the radiating electrons will re-absorb some of the photons and as a result the typical spectrum of a uniform synchrotron source will show a peak at $v_{\text {break }}$. Changes in the electron energy distribution and the decay of the magnetic field along the conical outflow forming the steady jet, imply that the critical frequency varies along the jet (Marscher 1995; Massi 2011).

In microquasars, the $v_{\text {break }}$ for the part of the steady jet closest to the engine (i.e., $v_{\text {break }_{1}}$ in Fig. 3) lies in the infrared (Russell et al. 2006), whereas in AGNs the observed turnover lies in the millimeter range (Marscher 1995). Observing at longer wavelengths than the infrared, i.e. in the radio band, gives rise to two results: a flat spectrum and the "core shift". Multi-wavelength observations result in the flat/inverted spectrum discussed in the introduction, which is typical of a steady jet. Imaging a steady jet at one observing frequency, $v_{\mathrm{obs}}$, gives rise to the effect known as "core shift", where the displacement from the center is a function of the observing frequency $v_{\mathrm{obs}}$. At $v_{\mathrm{obs}}$, the emission of the segment will dominate, whose spectrum peaks at that frequency plus small contributions from neighboring segments (see Fig. 1 in Markoff 2010). With the engine being close to the "infraredcore", it is clear that the $3.6 \mathrm{~cm}$-core can be displaced away from the orbit (as segment 2 is in Fig. 3 top-left). If a jet is pointing towards us (i.e., a microblazar as in Fig. 3 top-right), the core will be dominated by the approaching jet, because of Doppler boosting. If the jet is precessing, then the core will describe an ellipse (Fig. 3 top-right). A transient jet, in contrast, can undergo any shift from the center, depending on the velocity and the time elapsed from the transient. The peak of map E, even if displaced away from the others, and therefore likely affected by the approaching component of the transient jet, is at a position angle which is consistent that the other peaks. After $27 \mathrm{~d}$, the peak of run $\mathrm{I}$ is rather close to completing the cycle, i.e. to overlapping with the peak of the starting run A. The same occurs for the peak of run $\mathrm{J}$, that is $27 \mathrm{~d}$ after run $\mathrm{B}$, a peak that nearly overlaps with peak B. The peak of run J, $30 \mathrm{~d}$ after run A, is clearly displaced from the A peak. The period therefore seems to be in the range $27-28 \mathrm{~d}$.

\section{Conclusions and discussion}

Dhawan et al. (2006) suggested that their VLBA observations of LS I $+61^{\circ} 303$ could probe the cometary tail of the pulsar model and that the peaks of all images traced an erratic ellipse. In this paper, we have re-analyzed this data set and have shown that

1. The larger dynamic range of the self-calibrated maps reveals that the radio emission has in several images a double-sided structure.

2. Our fit analysis show that the peaks of the images at $8.4 \mathrm{GHz}$ trace a well-defined ellipse over a time interval of about $27-$ $28 \mathrm{~d}$.

The pulsar model explains neither the double-sided morphology nor the change from a double-sided to a one-sided structure. The microquasar model can explain them in terms of variable Doppler boosting, i.e., with a precessing jet. The $\mathrm{cm}$-core of a precessing steady jet pointing close to our line of sight, as in a microblazar, is expected to describe an ellipse during the precession. In the transient jet phase, there will be an additional shift due to the approaching jet component. We conclude therefore that the precession period is the time of about $27-28 \mathrm{~d}$ necessary to complete the ellipse. 

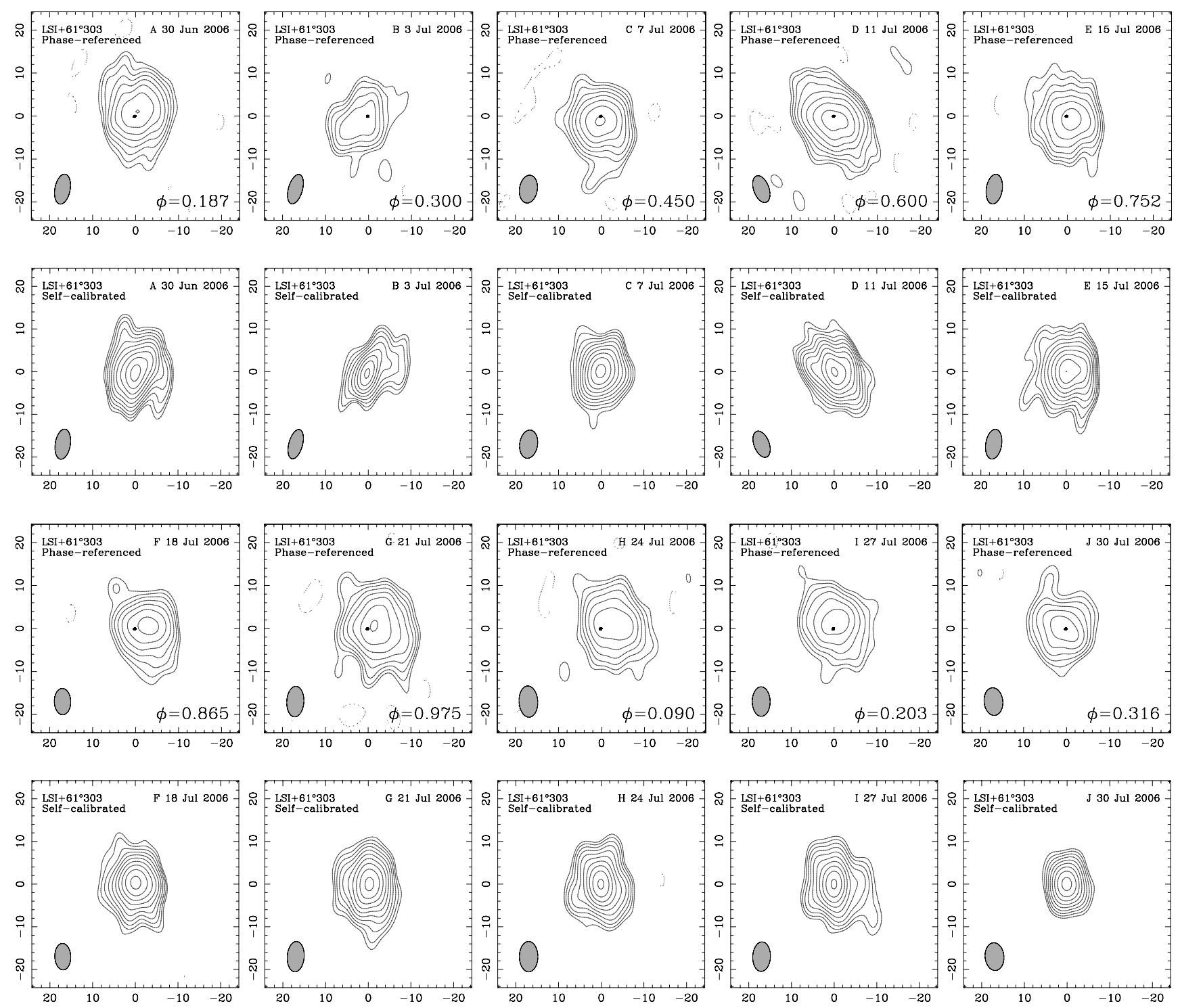

Fig. 5. Top: images of VLBA runs A-J at $13 \mathrm{~cm}(2.3 \mathrm{GHz})$ of LS I $+61^{\circ} 303$. For each run, two maps are presented, the phase referenced map and the self-calibrated map. Both maps are produced with uniform weight and the beam is 3.5-4.1 × 6.9-7.2. Contour levels for all maps are -4 , $4,5.66,8,11.3,16,22.6,32,45.2,64,90.5,128,181 \sigma$ (with $1 \sigma=0.4-0.6 \mathrm{mJy} /$ beam for phase-referenced maps, and $1 \sigma=0.3 \mathrm{mJy} / \mathrm{beam}$ for self-calibrated maps).

As shown in Fig. 3, owing to the core-shift effect, the core at $2.3 \mathrm{GHz}$ is expected to lie at a larger distance from the system center than at $8.4 \mathrm{GHz}$. However, because of the large scattering a proper astrometric analysis at $2.3 \mathrm{GHz}$ is impossible. Future observations should be done at high frequencies. By measuring core positions at many frequencies in the AGN M87, it was possible to determine the frequency dependence of the shift and find the converging location of all observing-wavelength dependent cores. The six frequency observation of M87 proved that $v_{\text {break }_{1}}$ is at $43 \mathrm{GHz}$ and that the $43 \mathrm{GHz}$-core is located within 23 Schwarzschild radii from the system center (Hada et al. 2011). Similar observations for LS I $+61^{\circ} 303$ would be particularly interesting not only to prove whether $v_{\text {break }_{1}}$ is in the infrared as expected for microquasars, but in particular to test whether the different cores at the different frequencies all trace ellipses with different sizes and all with the same period, as shown in the sketch of Fig. 3. An accurate determination of the period is mandatory to establish the physical process responsible for precession. Massi \& Zimmermann (2010) computed the precessional period for the accretion disk in LS I $+61^{\circ} 303$ under tidal forces of the $\mathrm{Be}$ star $\left(P_{\text {tidal-forces }}\right)$ and under the effect of frame dragging produced by the rotation of the compact object $\left(P_{\text {Lense-Thirring }}\right)$. By using those equations, we find that $P_{\text {tidal-forces }}$ of $28 \mathrm{~d}$ would require the unrealistic value for the size of the accretion disk of $R_{\text {out }}=0.5-0.8 \times 10^{13} \mathrm{~cm}$, i.e, nearly the semimajor axis, and can therefore be ruled out. In contrast, $P_{\text {Lense-Thirring }} \simeq 28 \mathrm{~d}$ would be realistic because it only implies a slow rotator with dimensionless spin parameter of $a_{*}=0.5 \times 10^{-3}$. However, whereas our study firmly rules out tidal forces, there could be other processes than frame dragging at work in LS I $+61^{\circ} 303$, which require a more accurate determination of the precessional period to be investigated. The orbital period is known with great accuracy and is $P=26.4960 \pm 0.0028 \mathrm{~d}$ (Gregory 2002), whereas our determination of the precessional period indicates a period of about 27-28 d. Therefore, it is important to establish, with future observations, whether the two periods, orbital and precessional ones, are indeed similar but still different. 
Acknowledgements. We thank the referee for his suggestions, which improved significantly the discussion and Lars Fuhrmann for useful conversations and comments to the manuscript. The Very Long Baseline Array is operated by the National Radio Astronomy Observatory, a facility of the National Science Foundation operated under cooperative agreement by Associated Universities, Inc. The Green Bank Interferometer is a facility of the National Science Foundation operated by the NRAO in support of NASA High Energy Astrophysics programs. The work of L. Zimmermann is partly supported by the German Excellence Initiative via the Bonn Cologne Graduate School. E.R. acknowledges partial support by the Spanish MICINN through grant AYA200913036-C02-02. E.R. was supported by the COST action MP0905 "Black Holes in a Violent Universe" through the short-term scientific mission (STSM) MP0905-300711-008633.

\section{References}

Bosch-Ramon, V., Paredes, J. M., Romero, G. E., \& Ribó, M. 2006, A\&A, 459, L25

Casares, J., Ribas, I., Paredes, J. M., Martí, J., \& Allende Prieto, C. 2005, MNRAS, 360, 1105

Cornwell, T., \& Fomalont, E. B. 1999, in Synthesis Imaging in Radio Astronomy II, ed. G. B. Taylor, C. L. Carilli, \& R. A. Perley, ASP Conf. Ser., 180, 187

Dhawan, V., Mioduszewski, A., \& Rupen, M. 2006, Proceedings of the VI Microquasar Workshop, 52.1

Dubus, G. 2006, A\&A, 456, 801

Fender, R. P., Belloni, T. M., \& Gallo, E. 2004, MNRAS, 355, 1105

Frail, D. A., \& Hjellming, R. M. 1991, AJ, 101, 2126

Gregory, P. C. 2002, ApJ, 575, 427

Hada, K., Doi, A., Kino, M., et al. 2011, Nature, 477, 185

Kaufman Bernadó, M. M., Romero, G. E., \& Mirabel, I. F. 2002, A\&A, 385, L10
Larwood, J. 1998, MNRAS, 299, L32

Lestrade, J.-F., Mutel, R. L., Preston, R. A., \& Phillips, R. B. 1985, Radio Stars, 116,275

Lestrade, J.-F., Preston, R. A., Jones, D. L., et al. 1999, A\&A, 344, 1014

Markoff, S. 2010, Lecture Notes in Physics, 794, 143

Marscher, A. P. 1995, PNAS, 92, 11439

Martí-Vidal, I. J. M., \& Marcaide, J. M. 2008, A\&A, 480, 289

Marti, J., \& Paredes, J. M. 1995, A\&A, 298, 151

Massi, M. 2007, The Multicolored Landscape of Compact Objects and Their Explosive Origins, AIP Conf. Proc., 924, 729

Massi, M. 2011, Mem. Soc. Astron. It., 82, 24

Massi, M., \& Kaufman Bernadó, M. 2009, ApJ, 702, 1179

Massi, M., \& Zimmermann, L. 2010, A\&A, 515, A82

Massi, M., Paredes, J. M., Estalella, R., \& Felli, M. 1993, A\&A, 269, 249

Massi, M., Ribó, M., Paredes, J. M., Peracaula, M., \& Estalella, R. 2001, A\&A, 376,217

Massi, M., Ribó, M, Paredes, J. M., et al. 2004, A\&A, 414, L1

Peracaula, M., Gabuzda, D. C., \& Taylor, A. R. 1998, A\&A, 330, 612

Paredes, J. M., Massi, M., Estalella, R., \& Peracaula, M. 1998, A\&A, 335, 539

Pradel, N., Charlot, P., \& Lestrade, J.-F. 2006, A\&A, 452, 1099

Rickett, B. J. 1990, ARA\&A, 28, 561

Romero, G. E., Okazaki, A. T., Orellana, M., \& Owocki, S. P. 2007, A\&A, 474, 15

Russell, D. M., Fender, R. P., Hynes, R. I., et al. 2006, MNRAS, 371, 1334

Shepherd, M. C. 1997, Astronomical Data Analysis Software and Systems VI, ed. G. Hunt, \& H. E. Payne, ASP Conf. Ser., 125, 77

Taylor, A. R., Kenny, H. T., Spencer, R. E., \& Tzioumis, A. 1992, ApJ, 395, 268

Taylor, A. R., Dougherty, S. M., Scott, W. K., Peracaula, M., \& Paredes, J. M. 2000, Proc. of Astrophysical Phenomena Revealed by Space VLBI, ed. H. Hirabayashi, P. G. Edwards, \& D. W. Murphy, 223 